\title{
BIOLOGICAL CONTROL OF GASTROINTESTINAL HELMINTHES OF LIVESTOCK
}

\author{
Dilşan BOYLU1 ${ }^{\text {, Hasan ÖNDER }}{ }^{2 *}$ \\ 1Ordu University, Agricultural Faculty, Department of Plant Protection, TR52200, Ordu, Turkey \\ 20ndokzu Mayıs University, Agricultural Faculty, Department of Animal Science, TR55139, Samsun, Turkey
}

Received: September 30, 2019; Accepted: December 03, 2019; Published: January 01, 2020

\begin{abstract}
Gastrointestinal helminths cause considerable production loss in ruminants globally and constraint to optimum livestock production. Extensively managed animals often host gastrointestinal parasites. Guidelines for sustainable control of parasites in sheep (SCOPS) have been produced in the UK which include recommendations for treatments to ewes. Traditionally control has been achieved through the application of broad spectrum anthelmintic; however, resistance is the major issue. In terms of parasitic nematodes in sheep, widespread resistance has been identified to the three available broad-spectrum anthelmintic classes with reports of increasing multi-class resistance was reported. Anthelmintic treatments can reach up to eight for lamb finishing. Resistance to anthelmintic can be reached to $88 \%$. The trend towards nonchemical parasite control methods (ecological, organic, green) farming of livestock. Biological control of nematode parasites of livestock is the rising issue of animal science. In this study, biological control of nematode parasites of livestock has been reviewed.
\end{abstract}

Keywords: Gastrointestinal helminthes, Livestock, Biological control

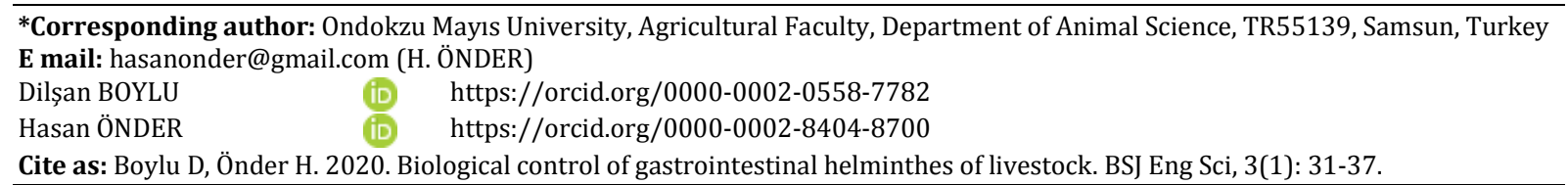

\section{Introduction}

Global food security and an increasing demand to produce more animal protein, due to growing human populations, will require the production of more food with more efficient land use, and reduced waste (Learmount et al., 2015). Gastrointestinal helminthes cause considerable production loss in livestock all over the world (Easton et al., 2018). Healthy adult ewes are mostly immune to infection with gastrointestinal parasites and as a result there is rarely a requirement for treatment with anthelmintic drugs. Around lambing, however, this immunity is lost, worm burdens increase, faecal egg counts (FECs) rise and farmers are more likely to treat the ewes to improve condition and also to reduce the potential contamination of pastures that will be grazed by naive lambs with little resilience. Anthelmintic treatments around lambing time have become common practice on UK farms as well as in other countries with a large sheep producing industry such as New Zealand. These treatments have been shown to be high risk for selection for anthelmintic resistance (Learmount et al., 2018). With increasing reports of anthelmintic resistance worldwide, control strategies for gastrointestinal nematodes that 
minimize the use of anthelmintic are of increasing importance. Research, particularly in the UK, Australia, South Africa and New Zealand, has resulted in a growing awareness of the problems of anthelmintic resistance and recognition of potential resistance-delaying strategies, which can be used on farms (Learmount et al., 2015). Control has traditionally been achieved through the frequent application of broad spectrum anthelmintics; however, this has led to the emergence and spread of resistance. Maintaining production and economic viability in the face of resistance is an increasing challenge (Easton et al., 2018).

The trend towards nonchemical parasite control methods (ecological, organic, green) farming of livestock. Biological control of nematode parasites of livestock is the rising issue of animal science. In this study, biological control of nematode parasites of livestock has been reviewed.

\section{Gastrointestinal Helminths}

There are many kind of gastrointestinal helminths such as Haemonchus contortus, Cysticercus tenuicollis, Teladorsagia circumcincta, Chabertia, Cooperia, Haemonchus, Nematodirus, Oesophagostomum, Teladorsagia, Trichostrongylus and Trichuris spp. cause considerable production loss in ruminants. The most common ones are;

\subsection{Haemonchus contortus}

Haemonchus contortus, also known as the barber's pole worm, is a very common parasite and one of the most pathogenic nematodes of ruminants. Adult worms attach to abomasal mucosa and feed on the blood. This parasite is responsible for anemia, oedema, and death of infected sheep and goats, mainly during summer in warm, humid climates. Females may lay over 10,000 eggs a day, which pass from the host animal in the faeces. After hatching from their eggs, $H$. contortus larvae molt several times, resulting in an L3 form that is infectious for the animals. The host ingests these larvae when grazing. The L4 larvae, formed after another molt, and adult worms suck blood in the abomasum of the animal, potentially giving rise to anaemia and oedema, which eventually can lead to death. The ova is yellowish in color. The egg (Figure 1) is about $70-85 \mu \mathrm{m}$ long by $44 \mu \mathrm{m}$ wide, and the early stages of cleavage contain between 16 and 32 cells. The adult female (Figure 2) is $18-30 \mathrm{~mm}$ long and is easily recognized by its trademark "barber pole" coloration. The red and white appearance is because $H$. contortus is a blood feeder, and the white ovaries can be seen coiled around the blood-filled intestine. The male adult worm is much smaller at 10-20 mm long, and displays the distinct feature of a well-developed copulatory bursa, containing an asymmetrical dorsal lobe and a Y-shaped dorsal ray.

The infection, called haemonchosis, causes large economic losses for farmers around the world, especially for those living in warmer climates. Anthelminthics are used to prevent and treat these, and other, worm infections, but resistance of the parasites against these chemicals is growing. Some breeds, such as the West African Dwarf goat and N'Dama cattle, are more resistant than other breeds to H. contortus (haemonchotolerance).

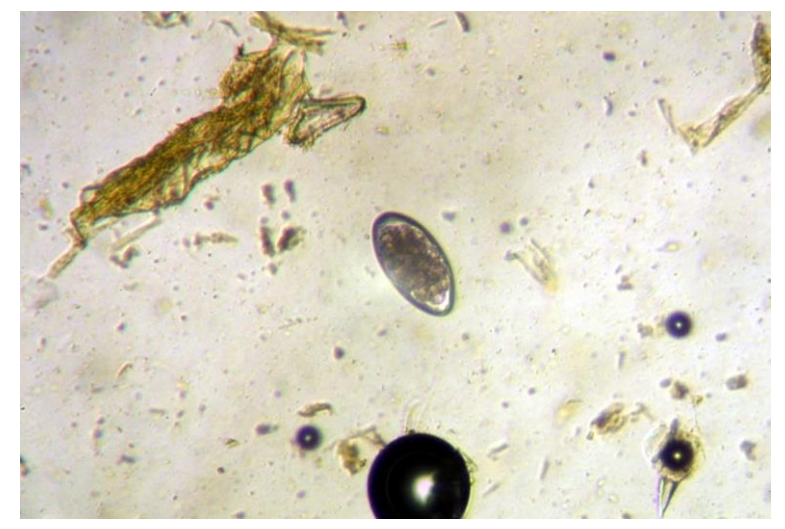

Figure 1. Haemonchus contortus egg.

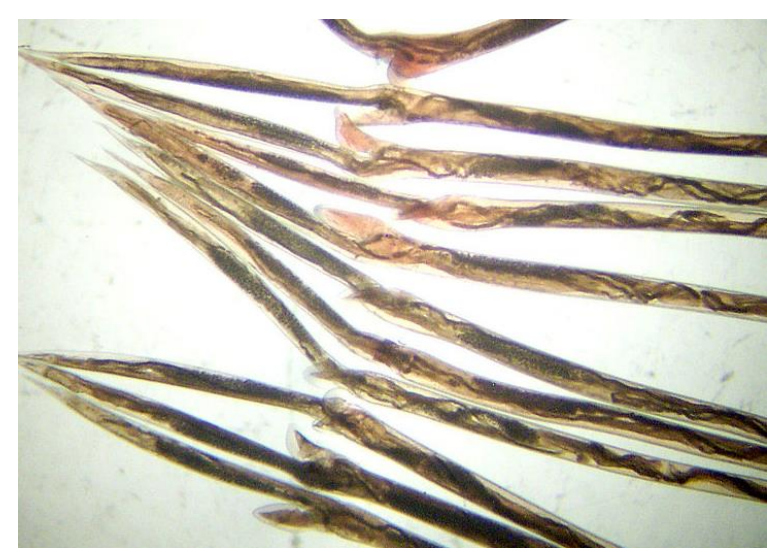

Figure 2. Haemonchus contortus adult females were taken from one sheep infected with a single strain of this worm species.

Clinical signs are largely due to blood loss. Sudden death may be the only observation in acute infection, while other common clinical signs include pallor, anemia, oedema, ill thrift, lethargy, and depression. The accumulation of fluid in the submandibular tissue, a phenomenon commonly called "bottle jaw", may be seen. Growth and production are significantly reduced.

Prophylactic anthelmintic treatment necessary to prevent infection in endemic regions, but wherever possible, a reduction on reliance on chemical treatment is warranted given the rapid rise of anthelmintic resistance (URL1).

\subsection{Cysticercus tenuicollis}

Cysticercus tenuicollis is the larval stage of the canine tapeworm Taenia hydatigena (Figure 3, Figure 4), the presence of which has been reported in wild and domestic ruminants all over the world. It is a common parasite of small ruminants in the north of Portugal. $C$. tenuicollis is generally seen attached to the omenta, the mesenteries or also found in the liver. In the ewe, tissue lesions have been associated with degenerative cysts or with oncosphere migrations. Infected carnivores 
eliminate T. hydatigena eggs with their faeces. Herbivores become infected with the eggs on account of having fed on contaminated pastures. Possible intermediate hosts for $C$. tenuicollis are squirrels, cattle, sheep, goats and other wild ruminants and also swines. After ingestion, the egg's shell is digested and the oncospheres become free to migrate through the intestinal walls, reaching the liver through the hepatic portal system. The oncospheres may remain in the liver or migrate to the omenta, the mesenteries and the serosal surface of the peritoneal cavity, where they attach and initiate post-oncospheral development (Payan-Carreira et al., 2008).

Like any other disease, the symptoms vary from its bodily location and when pertaining to the density of larvae. Many of the major symptoms are the result of inflammation during larval degeneration or a mass effect from the parasite. Neurocysticercosis is a serious form of cysticercosis. Common symptoms include chronic headaches and seizures. Other symptoms include: nausea, vomiting, vertigo, ataxia, confusion or other changes in mental health, behavioral abnormalities, progressive dementia, and focal neurologic signs (URL2).

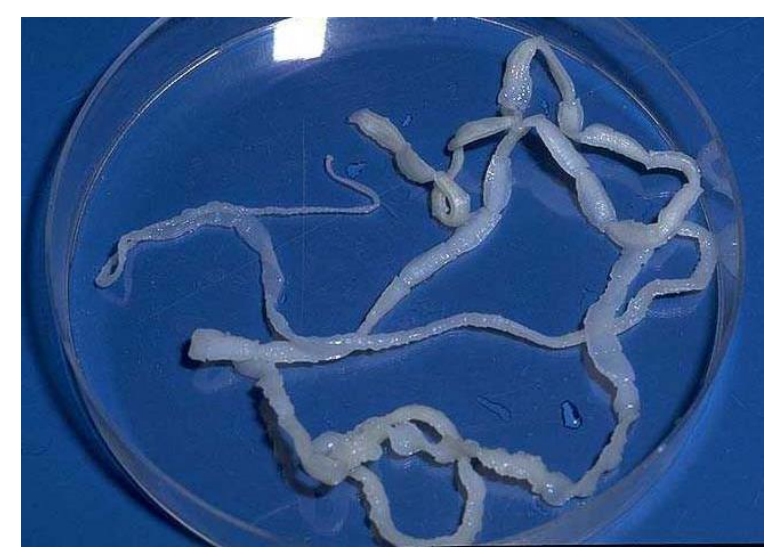

Figure 3. Taenia hydatigena in dog.

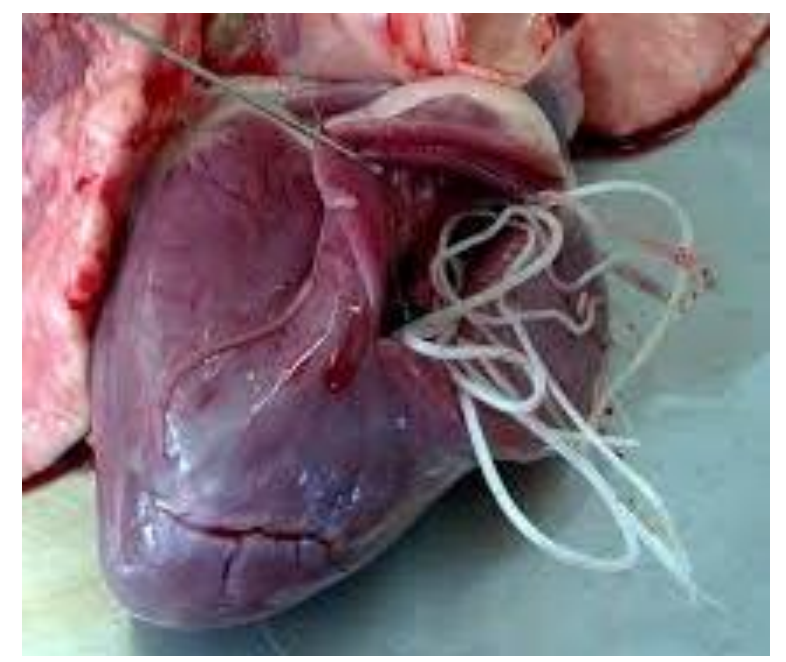

Figure 4. Taenia hydatigena in Sheep.
In the normal lifecycle of Taenia, cysticerci develop in the muscles of the intermediate hosts such as pigs, cattle, and sheep. In these animals, they do not cause severe symptoms. They are transmitted to humans when their infected meats are eaten. However, T. solium is unusual because its cysticerci can develop in humans. Due to accidental consumption of the eggs from contaminated foodstuffs, cycticerci in humans produce clinical symptoms. Thus, humans are accidental intermediate hosts (URL2).

Best prevention consists in preventing livestock feed (fresh pasture as well as hay, silage, and other stored feed) or water from being contaminated with dog feces that may contain tapeworm eggs. It must be considered that the eggs may remain infective in the feed after silage or other processing of hey or fodder (pelletizing, fermentation, etc.). The regular use of anthelmintics is not indicated for preventing horse or livestock infections with Cysticercus tenuicollis. There are reports that albendazole and praziquantel are effective, but only at dosis higher than the usual therapeutic ones, and results can be unreliable. There are so far no vaccines that would protect sheep or goats, other livestock or horses against Cysticercus tenuicollis. To learn more about vaccines against parasites of livestock. Biological control of Taenia tapeworms respectively Cysticercus tenuicollis (i.e. using its natural enemies) is so far not feasible (URL3).

\subsection{Teladorsagia circumcincta}

Teladorsagia circumcincta (Figure 5) is a nematode that is one of the most important parasites of sheep and goats. It was previously known as Ostertagia circumcincta and is colloquially known as the brown stomach worm. It is common in cool, temperate areas, such as south-eastern and south-western Australia and the United Kingdom. There is considerable variation among lambs and kids in susceptibility to infection. Much of the variation is genetic and influences the immune response. The parasite induces a type I hypersensitivy response which is responsible for the relative protein deficiency which is characteristic of severely infected animals. There are mechanistic mathematical models which can predict the course of infection. There are a variety of ways to control the infection and a combination of control measures is likely to provide the most effective and sustainable control.

Adults are slender with a short buccal cavity and are ruddy brown in colour. The average worm size varies considerably among sheep. Females range in size from 0.6 to $1.2 \mathrm{~cm}$ with males typically about $20 \%$ smaller.

The life cycle is relatively simple. Male and female adults breed on the abomasal surface. Eggs are passed in the faeces and develop through first (L1), second (L2) and third stage larvae (L3). Third stage larvae are unable to feed and are the infective stage. Following ingestion, third-stage larvae rapidly moult within 48 hours into fourth stage larvae (L4) which develop in the gastric glands. About 8 days after infection, the young adults 
emerge from the gastric glands, mature and breed. The prepatent period can be as short as 12 days. The number of eggs in the uterus of mature females can vary from less than 10 to more than 60 and is heavily dependent on the host immune response. The number of eggs produced per day by an adult female worm has been estimated as ranging from 0 to approximately 350 , with longer females laying more eggs.

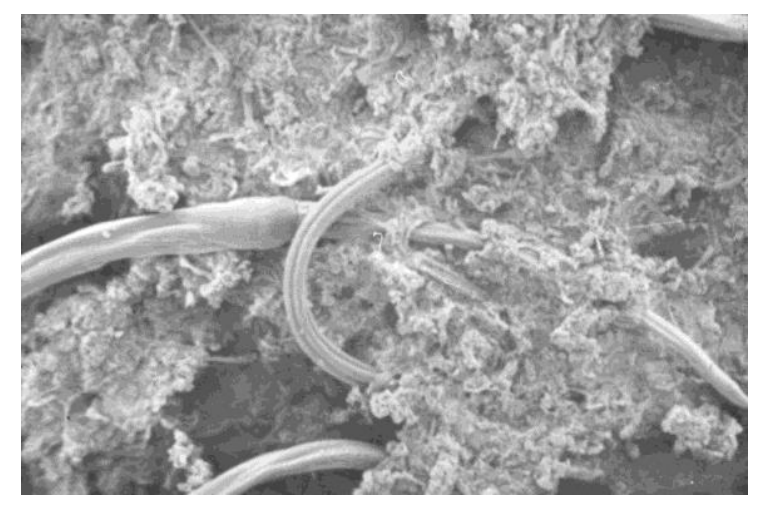

Figure 5. A scanning electron micrograph of Teladorsagia circumcincta.

It is responsible for considerable economic losses in sheep. It is believed to also cause severe losses in goats although there is a relative dearth of research in this species. In most countries, infection with third-stage larvae resumes in the spring and is triggered by the production of large numbers of eggs by periparturient females. The key trigger appears to be a relative protein deficiency in the ewe and the periparturient rise may be prevented by feeding supplementary protein. In the stereotypical pattern, egg production (assessed by faecal egg counts in the lambs) rises till midsummer then declines. The development of free-living nematodes is influenced by temperature and moisture and there is considerable variation within and among among years in the number of infective larvae available for ingestion. Consequently, the pattern of egg production during the year also varies among years. The number of infective larvae gradually dies down at the end of the grazing season when grass growth also declines. The end of the season varies among countries depending upon their climate and is also likely to vary from year to year within countries depending upon local weather but is poorly documented. For example, in Scotland, the season usually ends about late October.

There is considerable variation among animals in faecal egg counts and much of this variation is genetic in origin. The sources of variation are dynamic and their relative importance changes over the course of the year. Other sources of variation, apart from inherited effects include maternal and common environmental effects and effects specific to each individual, including variation in intake and non-additive genetic effects. The gender of the lamb, type of birth (single or twin), date of birth and intensity of early exposure to nematode infection have relatively minor but still important effects. The inevitable variation between the observed count and the true faecal egg count also contributes to the observed variation. Both the natural history of infection and the sources of variation among individuals are relatively well understood for $T$. circumcincta compared to most parasites.

Essentially all grazing animals are exposed to infection and most animals will carry some nematodes either as adults or arrested early fourth-stage larvae or both. For disease control, the aim is not to diagnose infection but to identify animals or flocks that are sufficiently heavily infected to show reduced production, decreased animal welfare or parasitic gastroenteritis. Heavily infected animals are relatively protein deficient. Clinical signs include reduced appetite, poor growth performance, weight loss and intermittent diarrhoea. In addition to clinical signs, faecal egg counts and the timing of infection are used to identify severely affected animals. However, lambs with very high numbers of worms produce very few eggs; there are strong density-dependent effects of worm number on egg production.

The methods to control nematode infection in livestock can be grouped into 6 categories: anthelmintic treatment, grazing management, biological control, nutritional supplementation, vaccination and genetic resistance. Each method has its advantages and disadvantages. Anthelmintic treatment usually involves treatment with one or more of five classes of broad spectrum drugs. Drugs: benzimidazoles, levamisole, macrocyclic lactones, amino acetonitrile derivatives and spiroindoles. Narrow spectrum drugs are also available. Drug formulations vary among countries and only some countries allow combinations of drugs to be used. Anthelmintic treatment is cheap, simple and effective but is threatened by the development of drug resistance in parasite populations. There are a number of strategies to mitigate the development of drug resistance including the use of combinations to delay the development of drug resistance in parasite populations. Drugs have been so cheap and effective that it was usually easier and cheaper to treat all animals in a population. However, not all individuals require treatment and best practice is now to target treatment on selected animals to delay the development of drug resistance. The criteria to decide which animals are to be treated and the size of the untreated population are matters of active research (URL4).

Biological control with spores of the fungus Duddingtonia flagrans has recently been licensed in Australia and can be very effective but requires regular, possibly daily, feeding (Waller and Thamsborg, 2004).

\section{Control of Gastrointestinal Helminths}

There are many kind of control approaches for gastrointestinal helminths such as chemical control, grazing management, feed - nutrients, feed antinutrients, ethnoveterinary remedies, genetics and 
breeding, vaccination and so on (Gray et al., 2012; Hoste and Torres-Acosta, 2011).

\section{Biological Control of Gastrointestinal Helminths}

Nematophagous fungi present themselves as natural enemies of gastrointestinal helminth parasites. They can be found in diverse environments and have been shown to be effective as biocontrol agents. In addition, an abundance of natural antagonists of helminths, including protozoa, bacteria, viruses, mites, beetles and fungi, have already been described as biological controllers. These organisms (nematophagous fungi) comprise different types of fungi and are cosmopolitan, occurring in natural soil, agricultural soils and in all types of decaying organic matter. In the environment these fungi are biologically very important, since they play a role in the recycling of carbon, nitrogen and other elements that originate from the degradation of the nematode (Braga and de Araujo, 2014). Nematophagous fungi are divided into three groups;

(1) Endoparasites are seen mainly as spores, but sometimes as chlamydospores (resistant spores) that are released at the time of the disintegration of the nematode. Their activity is infective, but they do not produce extensive fungal mycelium. Furthermore, they are not capable of growing in the soil, making them impossible to be proposed as inocula for the environmental control of a target nematode. Another point of great importance is their dependence on free water, which tends to be the main limiting factor for routine use under laboratory conditions as biological controllers of organisms (Stirling and West, 1991; Braga and de Araujo, 2014)

(2) Predators. This group includes the main species of nematophagous fungi - the genera Arthrobotrys, Duddingtonia and Monacrosporium. Their main feature is the production of traps and they have the advantage of greater potential for industrialization.

(3) Ovicidal or opportunistic fungi are those that parasitize eggs, cysts and nematode females. This group of fungi has been studied for a long time, and the first studies showed that these fungi would be promising in reducing populations of nematodes and helminths.

\subsection{Duddingtonia flagrans}

Currently, the work on biological control of nematode parasites of livestock is almost exclusively associated with the nematode-destroying microfungus Duddingtonia flagrans (Waller and Thamsborg, 2004). The microfungus has three very important attributes: (a) the ability to survive gut passage of livestock; (b) the propensity to grow rapidly in freshly deposited dung; and (iii) the possession of a voracious nematophagous capacity (Larsen, 1999). This fungus thus breaks the life cycle by capturing infective larval stages before they migrate from dung to pasture, where they would otherwise be acquired by grazing animals (Waller and Thamsborg, 2004). D. flagrans spore material; second, long-term field trials using $D$. flagrans have shown no adverse effects on the environment; and third, it has been established that $D$. flagrans is ubiquitous and that very close genetic similarity exists between isolates from all regions of the world (Waller, 2003; Waller and Thamsborg, 2004). Buzatti et al (2015) showed that D. flagrans reduced the number of infective larvae on fecal egg counts and the biological control with the predacious fungi $D$. flagrans is a promising free-living parasite regulator alternative to be use in livestock.

Duddingtonia flagrans (Figure 6) can be used against Barber's Pole Worm or Wire Worm (Haemonchus spp.), Black Scour Worm (Trichostrongylus spp.), Small Brown Stomach Worm (Teladosgia (Ostertagia) spp.), Medium Brown Stomach Worm (Ostertagia ostertagi), Nodule Worm (Oesophagostomum spp.), Hair Worm (Trichostrongylus spp.), Intestinal Worm (Cooperia spp.), Thin Necked Intestinal Worm (Nematodirus spp.) and Hook Worm (Bunostomum spp.) with great achievements (URL5).

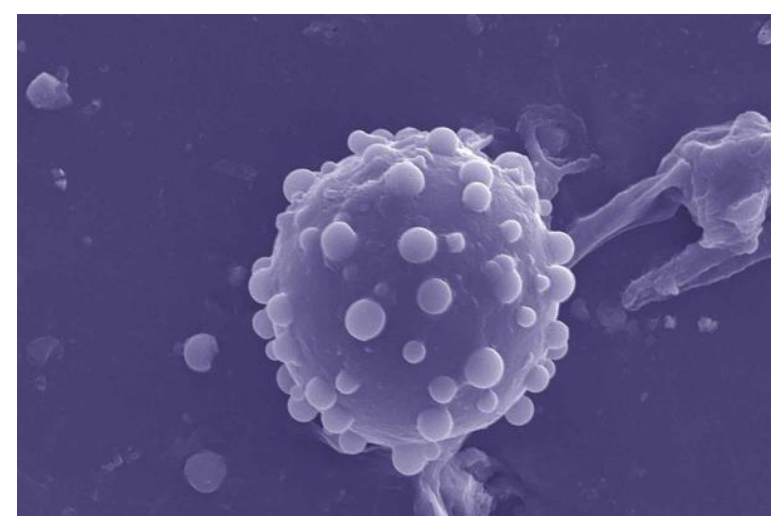

Figure 6. Duddingtonia flagrans.

\subsection{Arthrobotrys}

This genus is said to be 'classic' and was first described in the 19th century. It should also be mentioned that predatory fungi belonging to this group produce an extensive hyphal system along which are produced organelles capable of capturing nematodes. It is still valid to remember that among the predatory fungi that capture and kill nematodes, Arthrobotrys is probably one of the most important genera and certainly the most promising.

\subsection{Monacrosporium}

Fungi of the Monacrosporium genus are able to survive passage through the gastrointestinal tract of ruminants. This important premise regarding its activity, that it does not lose its predatory action, is of prime importance in the biological control of helminths. Monacrosporium also has proven action on gastrointestinal nematodes, which makes it a promising candidate to be used in the biological control of these organisms. The ability of $M$. thaumasium and $M$. sinense to survive passage through the gastrointestinal tract of domestic animals without 
losing predatory activity has been an important prerequisite for their use in biological control (Araújo et al. 1999; Braga and de Araujo, 2014).

\subsection{Ovicidal fungi}

These organisms, in addition to feeding on parasite eggs, cysts and females of nematodes and helminths, are saprophytic and, therefore, do not depend on the presence of the parasite in the soil for their survival and are thus easily cultured in the laboratory. This is a promising group of fungi to be employed in the biological control of helminths, mainly because they reduce levels of viable eggs in the soil (Braga and de Araujo, 2014).

\subsection{Pochonia}

This genus of ovicidal fungi is one of the most studied in the control of helminths that are potentially harmful to agriculture, and, currently, has also been standing out in combating their eggs. Again, it is noteworthy that experiments with this fungal genus include 'scientific enthusiasm' in their results, especially with regard to the effective control of nematode populations. $P$. chlamydosporia has been implicated as a suppressor of several genera of helminths, parasitizing their eggs by structures known as 'appressoria', formed from undifferentiated hyphae when in contact with these eggs (Braga and de Araujo, 2014).

\subsection{Paecilomyces}

Paecilomyces is a cosmopolitan filamentous fungus that exists worldwide and which inhabits soil, decaying plants, and food. In relation to the biological control of eggs from gastrointestinal parasites, research is advancing, but the main obstacle is the potential harmful 'element' that this genus and some strains can cause in man and animals (Braga and de Araujo, 2014).

\subsection{Predatory fungi}

Factors such as soil type, pasture, temperature, humidity, oxygen and predators determine a microclimate around the eggs and larvae on pasture, directly influencing the availability of infective larvae (L3). This microclimate is formed initially in the faecal matter, which is the source of larvae for the contamination of pastures. After transforming themselves into L3, larvae migrate to the adjacent pastures (within $90 \mathrm{~cm}$ of faecal matter and 15 $\mathrm{cm}$ at the base of the plants), where they can be ingested by the host. This migration usually occurs in the morning and late afternoon, when the light intensity is lower. The larvae often use water drops (such as dew) to migrate to pasture. Thus, the passage of nematophagous fungi through the gastrointestinal tract of domestic animals acts as an 'inundative biological control type', which consists of increasing concentrations of antagonist organisms already present in the soil (Braga and de Araujo, 2014).

\section{Results and Conclusion}

Biological control with fungi is a viable and safe alternative for animals, humans and the environment.
Gastrointestinal helminthes which caused considerable production loss in livestock can be controlled by biological methods instead of chemical control. More studies should be conducted to understand the effect and mechanisms of biological agents on helminths and effect of then on the environment.

\section{Conflict of interest}

The authors declare that there is no conflict of interest.

\section{Acknowledgements}

This study has been published in the Procceding book of International Congress on Domestic Animal Breeding Genetics and Husbandry-19 (ICABGEH-19).

\section{References}

Araújo JV, Stephano MA, Sampaio WM. 1999. Passage of nematodetrapping fungi through the gastrointestinal tract of calves. Vet Arh, 69: 69-78.

Braga FR, de Araujo JV. 2014. Nematophagous fungi for biological control of gastrointestinal nematodes in domestic animals. Appl Microbiol Biotechnol, 98: 71-82.

Buzatti A, de Paula Santos C, Fernandes MA, Yoshitani UY, Sprenger LK, dos Santos CD, Molento MB. 2015. Duddingtonia flagrans in the control of gastrointestinal nematodes of horses. Exp Parasitol, 159: 1-4.

Easton S, Pinchbeck GL, Bartley DJ, Hodgkinson JE, Matthews JB. 2018. A survey of experiences of UK cattle and sheep farmers with anthelmintic prescribers; Are best practice principles being deployed at farm level? Preventive Vet Med, 155: 27-37.

Gray GD, Connell JG, Phimphachanhvongsod V. 2012. Worms in smallholder livestock systems: Technologies and practices that make a difference. Veterinary Parasitology, 186: 124131.

Hoste H, Torres-Acosta JFJ. 2011. Non chemical control of helminths in ruminants: Adapting solutions for changing worms in a changing world. Veterinary Parasitology, 180: 144- 154.

Larsen M. 1999. Biological control of helminths. Int. J. Parasitol. 29: 139-146.

Learmount J, Gettinby G, Boughtflower V, Stephens N, Hartley K, Allanson P, Gutierrez AB, Perez D, Taylor M. 2015. Evaluation of 'best practice' (SCOPS) guidelines for nematodecontrol on commercial sheep farms in England and Wales. Veterinary Parasitology 207: 259-265.

Learmount J, Callaby C, Taylor M. 2018. An observational study of ewe treatments at lambing on early infection in lambs on UK sheep farms. Veterinary Parasitology, 253: 55-59.

Payan-Carreira R, Silva F, Rodrigues M, Dos Anjos Pires M. 2008. Cysticercus tenuicollisVesicle in Fetal Structures: Report of a Case. Reprod Dom Anim, 43: 764-766.

Stirling GR, West LM. 1991. Fungal parasites of root-knot nematodes eggs from tropical and sub tropical regions of Austrália. Australas Plant Pathol, 20: 149-154.

URL1: https://en.wikipedia.org/wiki/Haemonchus_contortus (access date: 05.08.2019).

URL2: https://en.wikipedia.org/wiki/Taenia_hydatigena (access date: 05.08.2019).

URL3:

https://parasitipedia.net/index.php?option=com_content\&vie 


\section{Black Sea Journal of Engineering and Science}

$\mathrm{w}=$ article $\& \mathrm{id}=2580$ (access date: 05.08.2019).

URL4:

https://en.wikipedia.org/wiki/Teladorsagia_circumcincta (access date: 05.08.2019).

URL5: https://www.youtube.com/watch?v=Jn_cxKs82aY (access date: 15.08.2019).

Waller PJ. 2003. Global perspectives on nematode parasite control in ruminant livestock: the need to adopt alternatives to chemotherapy, with the special emphasis on biological control. Animal Health Rev, 4: 35-43.

Waller PJ, Thamsborg SM. 2004. Nematode control in 'green' ruminant production systems. Trends in Parasitology, 20(10): 493-497. 